\title{
THE
}

$1-1-1983$

\section{Alternating linear-chain antiferromagnetism in copper nitrate $\mathrm{Cu}\left(\mathrm{NO}_{3}\right)_{2} .2 .5 \mathrm{H}_{2} \mathrm{O}$}

Jill C. Bonner

Simeon A. Friedberg

Hanako Kobayashi

Daniel L. Meier

Hendrik W. J. Blote

Follow this and additional works at: https://digitalcommons.uri.edu/phys_facpubs

Terms of Use

All rights reserved under copyright.

\section{Citation/Publisher Attribution}

Bonner, J. C., Friedberg, S. A., Kobayashi, H., Meier, D. L., \& Blöte, H. W. J. (1983). Alternating linear-chain antiferromagnetism in copper nitrate $\mathrm{Cu}\left(\mathrm{NO}_{3}\right)_{2} \cdot 2.5 \mathrm{H}_{2} \mathrm{O}$. Phys. Rev. B, 27(1), 248-260. doi: 10.1103/

PhysRevB.27.248

Available at: http://dx.doi.org/10.1103/PhysRevB.27.248

This Article is brought to you for free and open access by the Physics at DigitalCommons@URI. It has been accepted for inclusion in Physics Faculty Publications by an authorized administrator of DigitalCommons@URI. For more information, please contact digitalcommons-group@uri.edu. 


\title{
Alternating linear-chain antiferromagnetism in copper nitrate $\mathrm{Cu}\left(\mathrm{NO}_{3}\right)_{2} \cdot 2.5 \mathrm{H}_{2} \mathrm{O}$
}

\author{
Jill C. Bonner \\ Physics Department, University of Rhode Island, Kingston, Rhode Island 02881 \\ Simeon A. Friedberg, Hanako Kobayashi,* and Daniel L. Meier ${ }^{\dagger}$ \\ Physics Department, Carnegie-Mellon University, Schenley Park, Pittsburgh, Pennsylvania 15217
}

Hendrik W. J. Blöte $\ddagger$

Kamerlingh Onnes Laboratorium, University of Leiden, Nieuwsteeg 18, Leiden, Netherlands

(Received 21 July 1982)

\begin{abstract}
Current interest in the behavior of Heisenberg alternating antiferromagnetic quantum chains has been stimulated by the discovery of an unusual class of magnetoelastic spinPeierls systems. Copper nitrate, $\mathrm{Cu}\left(\mathrm{NO}_{3}\right)_{2} \cdot 2.5 \mathrm{H}_{2} \mathrm{O}$, does not display a spin-Peierls transition, but its dominant magnetic behavior is that of a strongly alternating antiferromagnetic chain with temperature-independent alternation. A remarkable, simultaneous fit is demonstrated between theoretical studies and a wide variety of zero- (low-) field experimental measurements, including susceptibility, magnetization, and specific heat. The fitting parameters are $\alpha$ (degree of alternation) $=0.27, J_{1} / k=2.58 \mathrm{~K}, g_{b}=2.31$, and $g_{\perp}=2.11$. Slight systematic discrepancies are attributed to weak interchain coupling. Theoretical studies also predict a rich variety of behavior in high fields, particularly in the region involving the lower and upper critical fields, $H_{c_{1}}=28 \mathrm{kOe}$ and $H_{c_{2}}=44 \mathrm{kOe}$. Experimental specific-heat measurements at $H=28.2$ and 35.7 kOe show quantitative agreement with theory in this interesting parameter region. The fitting parameters are the same as for zero field and, again, small discrepancies between theory and experiment may be attributed to interchain coupling. The exceptional magnetic characterization of copper nitrate suggests its use for further experimental study in the vicinity of the high-field ordering region.
\end{abstract}

\section{INTRODUCTION}

Alternating linear magnetic chains have lately been the subject of increasing interest. Originally studied primarily by physical chemists for their relation to organic free radicals and spin-exciton theory, ${ }^{1}$ they have more recently aroused interest among physicists in connection with spin-Peierls theory. ${ }^{2}$ Coordination chemists are currently interested in homologous families of alternating-chain systems because of information they may provide on variation of exchange constant with bond length, bond angle, and possibly other factors. ${ }^{3,4}$

The Hamiltonian for an alternating Heisenberg linear chain may be written

$$
H=-2 \sum_{i=1}^{N / 2}\left(J_{1} \overrightarrow{\mathrm{S}}_{2 i-1} \cdot \overrightarrow{\mathrm{S}}_{2 i}+J_{2} \overrightarrow{\mathrm{S}}_{2 i} \cdot \overrightarrow{\mathrm{S}}_{2 i+1}\right),
$$

representing a chain where pairs of spins are alternately coupled by strong and weak bonds. Introducing an alternation parameter $\alpha$, Eq. (1) may conveniently be written

$$
H=-2 J_{1} \sum_{i=1}^{N / 2}\left(\overrightarrow{\mathbf{S}}_{2 i-1} \cdot \overrightarrow{\mathbf{S}}_{2 i}+\alpha \overrightarrow{\mathbf{S}}_{2 i} \cdot \overrightarrow{\mathbf{S}}_{2 i+1}\right),
$$

where $\alpha=J_{2} / J_{1}$. When $\alpha=1$, the familiar Heisenberg linear chain is obtained (hence we call $\alpha=1$ the uniform limit), whereas when $\alpha=0$ the system breaks up into an assembly of noninteracting spin pairs or dimers. All known experimental alternating-chain systems have isotropic (Heisenberg) spin symmetry, a spin value of one-half, and dominant antiferromagnetic interactions, i.e., $J_{1}=-\left|J_{1}\right| . \quad \mathrm{Cu}\left(\mathrm{NO}_{3}\right)_{2} \cdot 2.5 \mathrm{H}_{2} \mathrm{O}$, which we shall refer to simply as "copper nitrate," was the first inorganic alternating-chain system to be investigated experimentally, and is at present the best characterized. $\mathrm{Cu}\left(\mathrm{NO}_{3}\right)_{2} \cdot 2.5 \mathrm{H}_{2} \mathrm{O}$ is a strongly alternating (or dimerized) system, with $\alpha \sim 0.3$. Other alternating systems span the alternation range and include a number of organic free radicals ${ }^{1}$ as well as the copper compounds copper bromide $\mathrm{N}$ methylimidazole [Cu(NMI) ${ }_{2} \mathrm{Br}_{2}, \alpha \sim 0.4$ (Ref. 4)], copper chloride $N$-methylpyridine $\left[\mathrm{Cu}(4 \mathrm{MP})_{2} \mathrm{Cl}_{2}\right.$, $\alpha \sim 0.7]$, catena-hexanonebis (thiosemicarbazato) 
copper (II) [Cu-HTS, $\alpha \sim 0.9$ (Ref. 3)], and catenaoctanedionebis (thiosemicarbazato) copper (II) [CuOTS, $\alpha \sim 0.9$ (Ref. 3)].

Spin-Peierls systems are rather different. Above the spin-Peierls transition $\left(T_{\mathrm{sp}}\right)$ they behave as an assembly of uniform Heisenberg chains. Below the (second-order) $T_{\text {sp }}$, progressive spin-lattice dimerization distortion effectively gives rise to an assembly of alternating chains such that the degree of alternation $\alpha$ is temperature dependent. $\alpha$ increases from zero at $T_{\mathrm{sp}}$ to a value of $\alpha_{\max }$ as $T$ decreases to zero, where for all known systems, $\alpha_{\max } \geq 0.7$.

The unusual properties of copper nitrate were first observed at Carnegie-Mellon University in a series of susceptibility, ${ }^{5}$ magnetization, ${ }^{6}$ and specific-heat ${ }^{7}$ studies on both powder and single-crystal samples in low fields. Other early work included $\mathrm{x}$-ray studies, ${ }^{8,9}$ proton resonance, ${ }^{10}$ and magnetocaloric measurements. ${ }^{11}$ Alternating-chain character in copper nitrate is not obvious from its $\mathrm{x}$-ray structural analysis alone and was established from various pieces of evidence. That character has only recently been confirmed fairly directly by neutron scattering techniques. ${ }^{12}$ Early magnetic studies indicated that a model of noninteracting spin-pairs (dimers) gave a good first approximation to the properties of this substance. ${ }^{5-7}$ However, systematic discrepancies between theory and experiment of about $5 \%$ remained, for example, in the height of the specificheat maximum. ${ }^{13}$ Similarly the magnetization isotherms cannot be fitted to better than about $5 \%$ (Ref. 6) with the dimer model. A weak antiferromagnetic interdimer mean-field interaction considerably improved the fit to the magnetization data, thus establishing the importance of interdimer coupling. ${ }^{6}$ A mean-field interaction of this type does not, however, remove the zero-field specific-heat discrepancy. ${ }^{14,15}$ Crystal structure determinations $\mathbf{s}^{8,9}$ suggested two possible interdimer coupling schemes, both essentially one-dimensional in nature. ${ }^{15,16}$ In the first model the dimers are linked into zig-zag alternating chains, and in the second model the dimers form the "rungs" of a ladder, the weaker interdimer interactions forming the long sides of the ladder (ladder model). $X$-band EPR measurements confirmed the plausibility of these two models. ${ }^{17}$ Accordingly, exact finite-cluster calculations were performed, primarily on alternating chains and ladder models of four and six spins, and the results fitted to the available zero- and low-field data. ${ }^{15}$ An excellent, simultaneous fit was obtained for all data using a set of $J$ and $g$ values consistent with independent experimental determinations. This indicates the basic validity of the one-dimensional (1D) models and was made possible by the rapid convergence of the calculations in zero field. In fact a six-spin clus- ter is very close to the corresponding model in the thermodynamic limit. What these finite-cluster studies did not do was distinguish between alternating chain and ladder models.

Magnetic, thermal, and proton resonance studies at the Kamerlingh Onnes Laboratorium by van Tol et al. .8-22 $^{2}$ and Haseda et al. in Kyoto and Osa$\mathrm{ka}^{23,24}$ were subsequently extended by Diederix and co-workers. ${ }^{25-30}$ By a careful analysis of the angular dependence of the proton resonance splitting, Diederix et $a{ }^{28}{ }^{28}$ concluded that the ladder model could be unambiguously ruled out, whereas good agreement between observed and calculated alternating-chain curves was obtained. However, an uncertainty remained as to whether the alternating pathways occurred in the $a-c$ or $a-b$ planes. This ambiguity was finally resolved by neutron scattering studies on deuterated copper nitrate ${ }^{12}$ which confirmed the correctness of the alternating-chain description and, further, established the dominant alternating pathway to lie in the $a-c$ plane. Diederix et al. also proposed ${ }^{28}$ two weak interchain interactions, one of which tends to link adjacent chains within the $a-c$ plane into two-dimensional (2D) sheets while the other, along the $b$ direction, leads to a coupled three-dimensional (3D) array. They concluded that exchange coupling along the $b$ axis was ferromagnetic but could not determine the sign of interchain interaction in the $a-c$ plane. The neutron scattering studies ${ }^{12}$ of the ordered structure confirm that the $b$-axis coupling is ferromagnetic and show that interaction between chains in the $a-c$ plane is of antiferromagnetic sign.

Interchain coupling leads to the establishment of long-range order in copper nitrate at nonzero temperature only in a nonzero applied magnetic field. Such ordering has been observed below $\sim 0.17 \mathrm{~K}$, with a field along the $b$ axis, by several methods including proton resonance, specific-heat, magnetocaloric, and neutron scattering studies. The transition temperature is strongly field dependent and is nonzero only for fields between $\sim 28$ and 44 kOe. The ordered phase is quite unusual. It is analogous to the spin-flop phase of a weakly anisotropic uniaxial antiferromagnet in that the spins tend to align antiferromagnetically in the $a-c$ plane if the field is applied in the $b$ direction. An important step in the experimental study of field-induced spin ordering in copper nitrate was the observation of unusual magnetocaloric behavior above $\sim 0.18 \mathrm{~K}$ by Amaya et al. ${ }^{11,31}$ These results stimulated theoretical studies $^{32,33,34}$ of long-range ordering in systems of exchange-coupled ion pairs with interpair interactions and were initially thought to exemplify such behavior. It was soon pointed out, ${ }^{15}$ however, that the dominant interpair interactions in 
$\mathrm{Cu}\left(\mathrm{NO}_{3}\right)_{2} \cdot 2.5 \mathrm{H}_{2} \mathrm{O}$ probably created $1 \mathrm{D}$ arrays of pairs in which short-range order would be pronounced well above the temperature at which weaker interchain coupling would produce long-range order. It was also noted that the results of Amaya et al. could be explained by such short-range order alone. Numerous subsequent theoretical ${ }^{35-37,16}$ and experimental ${ }^{18,19,22,27-29}$ investigations have confirmed these conclusions. However, the interesting magnetic cooling behavior of $\mathrm{Cu}\left(\mathrm{NO}_{3}\right)_{2} \cdot 2.5 \mathrm{H}_{2} \mathrm{O}$ is not our present concern and will not be discussed further here.

The small-cluster studies stimulated exact finitechain calculations of $N=2,4,6,8,10$, and 12 spins. ${ }^{29,38}$ Convergence properties were good enough to allow extrapolation to the thermodynamic limit over a field range of zero to about $50 \mathrm{kOe}$. In Secs. III and IV of this paper zero- and high-field data, respectively, will be analyzed in terms of an alternating chain with $\alpha=0.27$. This agrees with the $\alpha$ value deduced by Diederix et al. from high-field measurements only. In all cases remarkable agreement is obtained between theory and experiment, residual discrepancies being primarily attributable to neglect of interchain coupling. The theoretical aspects of alternating-chain systems will be discussed in Sec. II, and Sec. V will be a review of the significance of the present results; future experimental and theoretical investigations on this interesting system are outlined in Sec. IV.

\section{THEORETICAL PROPERTIES OF ALTERNATING CHAINS}

Until recently 1D systems with lattice (bond) alternation have been less intensively studied than the corresponding uniform systems or spin-anisotropic systems. The Heisenberg alternating, spin- $\frac{1}{2}$, antiferromagnetic chain has some interesting properties that might not, initially, be expected.

The first unusual feature is that the powerful analytic technique based on the Bethe ansatz which solves the general, anisotropic uniform-exchange quantum spin chain, fails for the Heisenberg alternating spin chain. Hence theoretical treatments to date have all been approximate, the most reliable being extrapolation techniques based on exact calculations on finite chains of $2,4,6,8$, and 10 spins by

$$
g \mu_{B} H_{c_{2}}=2 J_{1}(1+\alpha) \text { (exact), }
$$

for the Ising-Heisenberg AFM:

$$
g \mu_{B} H_{c_{2}}=2 J(1+\gamma) \quad \text { (exact), }
$$

Duffy and Barr. ${ }^{39}$ The approach has recently been extended to include chains of 12 spins, and to a more extensive range of alternation $(\alpha)$ values, particularly near the uniform limit. ${ }^{38}$

The extended calculations ${ }^{38}$ show, rather conclusively, that an excitation energy gap exists between a nondegenerate singlet ground state and a continuum of excited triplet states (as predicted by spin-exciton theory ${ }^{1}$ ). Duffy and Barr concluded from their calculations that this gap extends from the dimer limit $(\alpha=0)$ to $\alpha \sim 0.5$ or 0.6 , but they did not care to commit themselves for higher $\alpha$ values. The more recent demonstration of the existence of a gap for all nonzero alternation has caused some surprise ${ }^{40}$ since intuition suggests that a gapless "acoustic" mode should always be present in the case of the Heisenberg Hamiltonian because of the complete rotational symmetry in spin space. However, further investigation reveals that a gapless mode need not necessarily be present when the ground state is nondegenerate, as is the case here. It might be noted, however, that the ground state of the corresponding alternating Heisenberg ferromagnet is $\mathrm{N}$-fold degenerate and a gapless mode does indeed exist in this case for all nonzero alternation.

The presence of an excitation energy gap gives rise to some qualitative similarities ${ }^{41}$ between an alternating Heisenberg system and an anisotropic Ising-Heisenberg (easy-axis anisotropy) system which also has an energy gap for all nonzero anisotropy, as is known rigorously. ${ }^{42}$ The Hamiltonian for this system is

$$
H=2|J| \sum_{i=1}^{N}\left[S_{i}^{z} S_{i+1}^{z}+\gamma\left(S_{i}^{x} S_{i+1}^{x}+S_{i}^{y} S_{i+1}^{y}\right)\right],
$$

where $\gamma$ is a spin-anisotropy parameter which varies between $\gamma=0$ (Ising model) and $\gamma=1$ (Heisenberg model). The application of a magnetic field, i.e., inclusion of a Zeeman term $\left(-g \mu_{B} H \sum_{i} S_{i}^{z}\right)$, depresses the excited levels relative to the ground state, eliminating the gap at a certain $T=0$ critical field, $H_{c_{1}}$. At a higher $T=0$ field, $H_{c_{2}}$, the effect of the Zeeman term is to make the fully aligned ferro-type state the ground state, with a second energy gap opening up, proportional to the applied field, between spin-aligned ground state and other states. For 1D systems the critical fields are as follows. For the alternating Heisenberg antiferromagnet (AFM):

for the Ising-Heisenberg AFM: 


$$
g \mu_{B} H_{c_{1}}=\frac{J}{2} \sinh \phi \sum_{n=-\infty}^{\infty} \frac{(-1)^{n}}{\cosh n \phi} \frac{\pi J \sinh \phi}{2 \phi}=\sum_{n=-\infty}^{\infty} \frac{1}{\cosh \left\{[(2 n+1) / 2]\left(\pi^{2} / \phi\right)\right\}},
$$

where $\cosh \phi=1 / \gamma$, and for the alternating Heisenberg AFM:

$$
g \mu_{B} H_{c_{1}}=J_{1}\left[2-\gamma-\frac{3}{4} \alpha^{2}+\frac{1}{16} \alpha^{3}+O\left(\alpha^{4}\right)\right],
$$

where $\alpha=J_{2} / J_{1}$.

This defines several field regions such that the functional dependence on temperature of the thermodynamic properties varies from region to region in a striking way. In the case of the specific heat, for example, we have the following behavior:

$$
\text { (a) } 0 \leq H<H_{c_{1}}: C_{H} \sim T^{-3 / 2} \exp \left(-\Delta E_{1} / k T\right) \text {, }
$$

where $\Delta E_{1}(H)$ is the energy gap for $0 \leq H<H_{c_{1}}$,

$$
\begin{aligned}
& \text { (b) } H_{c_{2}}<H: C_{H} \sim T^{-3 / 2} \exp \left(-\Delta E_{2} / k T\right) \text {, } \\
& \text { (c) } H_{c_{1}}<H<H_{c_{2}}: C_{H} \sim A(H) T, \\
& \text { (d) } H=H_{c_{1}}: C_{H} \sim B_{1} T^{1 / 2}, \\
& \text { (e) } H=H_{c_{2}}: C_{H} \sim B_{2} T^{1 / 2} .
\end{aligned}
$$

This pattern of behavior holds for both alternating and spin-anisotropic systems. For the spinanisotropic system, some analytic results and numerical results are available for the constants $A$ and $B .{ }^{41}$ Only approximate spin-wave calculations are available for the alternating antiferromagnet.

In regions (a) and (b) the susceptibility $\chi$ goes exponentially to zero as $T \rightarrow 0$ as $T^{1 / 2} \exp \left(-\Delta E_{1,2} / k T\right)$ on account of the energy gap. In the continuum region (c), where $H_{c_{1}}<H<H_{c_{2}}, \chi$ tends to a constant nonzero value $\chi_{0}(H)$ as $T \rightarrow 0$. Finally, at the special fields, $H_{c_{1}}$ and $H_{c_{2}}, \chi$ diverges as $T^{1 / 2}$. A discussion, together with experimental results, is given in Diederix et al. ${ }^{29}$ Clearly this fascinating variety of behavior is worthy of experimental study, and some additional results are presented in Sec. IV.

Of course, in real systems, we are not dealing with the ideal $1 \mathrm{D}$ case, but must take account of weak interchain interactions, i.e., deal with quasi-1D systems. One important difference between quasi-1D alternating Heisenberg antiferromagnets and quasi1D spin-anisotropic antiferromagnets lies in the $T>0$ phase behavior. This is illustrated, schematically, in Figs. 1(a) and 1(b). Figure 1(b) is nothing other than the quasi-1D version of the familiar $H_{\|}-T$ phase diagram of a spin-flop antiferromagnet. At low fields an ordered antiferromagnetic phase is present, with a first-order transition to a spin-flop phase at higher fields, between $T=0$ critical fields $H_{c_{1}}$ and $H_{c_{2}}$. A prominent feature of a quasi-1D spin-flop antiferromagnet is the pronounced bicritical point $B$.

For the quasi-1D alternating antiferromagnet of Fig. 1(a), the phase diagram is strikingly different. The low-field ordered antiferromagnetic phase is absent, leaving only a spin-flop-type phase for $H_{c_{1}}<H<H_{c_{2}}$. Simple, approximate theoretical treatments, such as mean-field theory and spin-wave theory, ${ }^{42}$ indicate that the boundary of this isolated high-field phase should be of second order, in agreement with existing experimental results to be discussed later. Since the alternating Heisenberg chain cannot be solved exactly, let alone the problem of interacting alternating chains, the above phase

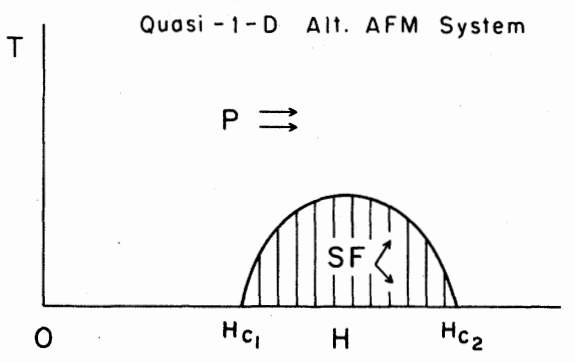

(a)

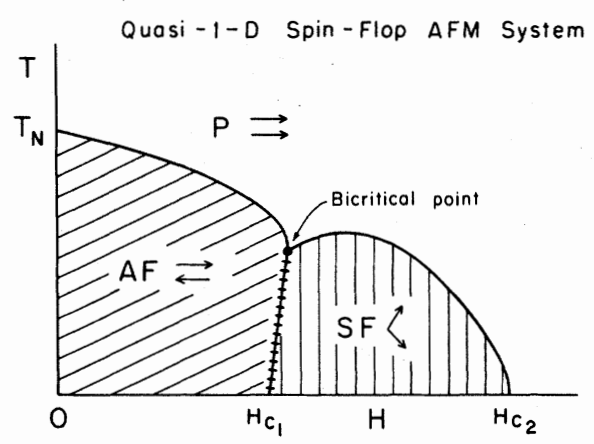

(b)

FIG. 1. Schematic phase diagram for quasi-onedimensional antiferromagnets in applied magnetic fields: (a) weakly interacting alternating Heisenberg AFM chains and (b) weakly interacting, weakly anisotropic AFM chains with $H_{\|}$preferred spin axis. 
behavior cannot be claimed to be rigorously correct. Nevertheless, the accumulated supporting evidence is strong, in which case we have the intriguing situation of a 3D system which shows no ordered phase behavior in zero field, but only in high fields. We might note that the well-known condition or test for the existence of an ordered phase in a given system due to Mermin and Wagner. ${ }^{44,45}$ applies only to zero field, and, used naively, would give misleading results for this system.

To lend support to the possibility of unusual phase behavior of the type adduced for weakly coupled alternating antiferromagnetic chains, we note the existence of rigorously solvable models which show similar behavior in the sense of displaying phase transitions in nonzero applied field and not in zero field. ${ }^{46-48}$ Some feeling for the presence of an antiferromagnetic ordered phase in the anisotropic system and not in the alternating system, may come from the fact that the ground state of the ideal 1D anisotropic system is ordered at $T=0,{ }^{49-51}$ whereas the ideal alternating antiferromagnetic chain has a nonordered nondegenerate singlet ground state. ${ }^{52}$ Although the dominant magnetic structure of copper nitrate is one of strongly alternating Heisenberg antiferromagnetic chains with weaker antiferromagnetic interchain coupling in two-dimensional layers, even weaker interlayer interactions, ferromagnetic in type, are present. (See discussion in Secs. I and IV.) This raises the interesting possibility that the weak, residual, but competing ferromagnetic interactions may influence the nature of the high-field phase transition, at least at very low temperatures. For example, the very-low-temperature portions of the phase boundary may become of first order, giving rise to a pair of tricritical points, or other novel phase behavior may occur. Existing experimental data, such as proton resonance results, do not reveal such features but may not have been carried down to sufficiently low temperatures.

Let us now return to a discussion of the theoretical properties of alternating chains in zero (or low) fields since this forms a major portion of the present study. The diagonalization and extrapolation procedures used to infer the behavior of the system in the thermodynamic limit from exact finite-chain calculations are rather well known. ${ }^{49,29,39}$ In the case of the specific heat, for example, the convergence for finite periodic alternating systems (for $\alpha=0.3$ ) is so rapid that the curves for $N=8,10$, and 12 are indistinguishable, at least on the scale of Fig. 2. For $\alpha$ values as close as this to the dimer limit, rapid convergence is also the case for the susceptibility (not shown). By contrast, near the uniform limit, convergence is slow, as is shown in Fig. 3 for the specific heat at $\alpha=0.8$. We briefly men-

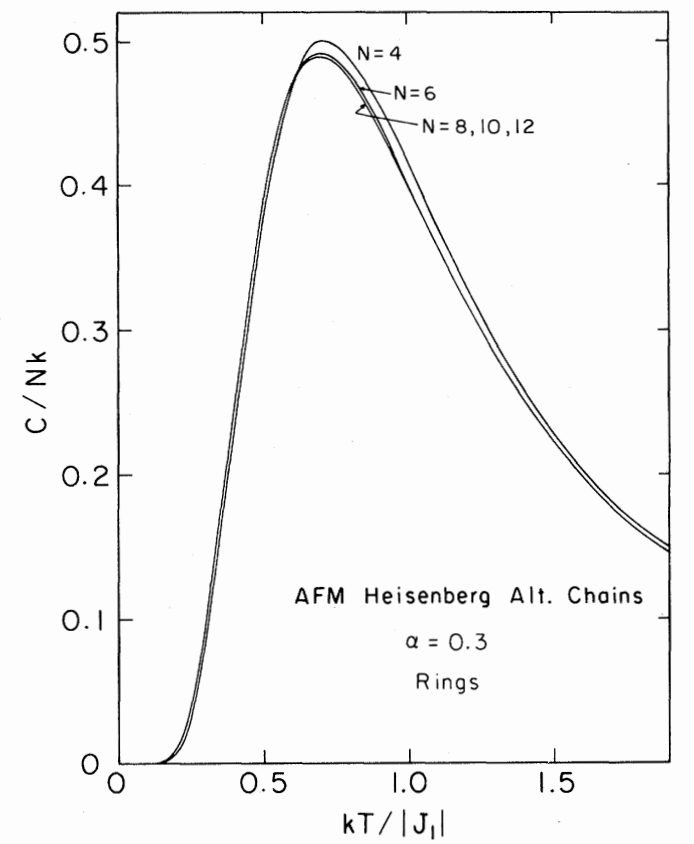

FIG. 2. Zero-field finite-chain specific-heat calculations (periodic boundary conditions) for AFM Heisenberg chains with large alternation. Curves for $N=4,6,8,10$, and 12 are shown. Note the rapid convergence with $N$ which makes the curves for $N=8,10$ and 12 indistinguishable on the scale of this figure.

tion that very recent work, which maps the alternating Heisenberg antiferromagnet into a 2D classical Ashkin-Teller or staggered eight-vertex model at marginal $q$-component dimensionality, ${ }^{53,54}$ gives us insight into this problem. The implication is that logarithmic correction terms will be present which will slow convergence near the uniform limit. ${ }^{38}$ Fortunately, this convergence problem is not relevant to a study of copper nitrate.

At this point, the reader may be interested in a perspective of how the specific heat varies as a function of alternation in zero field. This is shown in Fig. 4 (which is an extended version of Fig. 10 of Ref. 39 using the new $N=12$ data). The overall trend is that the maximum drops in magnitude and moves to higher temperatures. A corresponding display plot to Fig. 4 for the zero-field susceptibility has already been published in relation to spin-Peierls theory. ${ }^{55}$ Unfortunately, the rapid convergence for low $\alpha$ values in zero field does not extend to the anomalous field region where $H_{c_{1}} \leq H \leq H_{c_{2}}$, at least, not for low temperatures. This point is further discussed in Sec. IV.

The effect on the energy spectrum of alternating chains near the dimer limit of an applied field has been discussed by Diederix et al. ${ }^{29}$ The ground state 


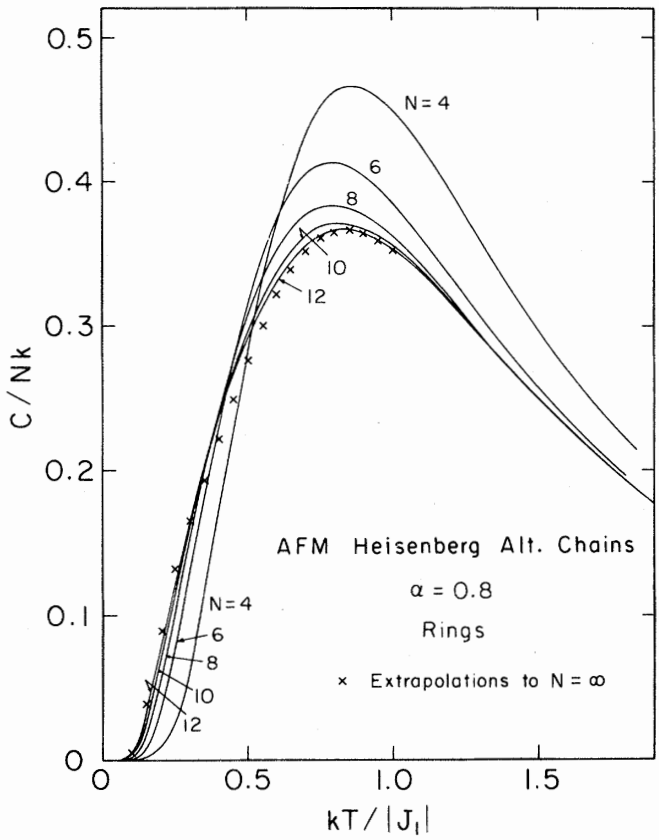

FIG. 3. Zero-field finite-chain specific-heat calculations (periodic boundary conditions) for AFM Heisenberg alternating chains close to the uniform limit. Solid curves denote results for $N=4,6,8,10$, and 12 , and crosses represent extrapolations to the thermodynamic limit. Note that convergence near the uniform limit is much slower than convergence near the dimer light.

remains a nondegenerate singlet, ${ }^{53}$ but the degenerate excited states (in the dimer limit) are split into a series of exciton bands by the interdimer interaction. A fuller discussion of the details of the energy spectra of alternating chains for general alternation is given in Ref. 38. In the anomalous field region the levels again "bunch up" into a set of energy bands (see Fig. 1 of Ref. 29) leading to unusual behavior of, say, the specific heat.

Finally, we conclude our theoretical survey of the properties of ideal and quasi-1D Heisenberg alternating antiferromagnetic chains by considering the $T=0$ magnetization curves. They are shown in Fig. 5 as a function of $\alpha^{56-58}$ For $0<\alpha<1$, the two critical fields, $H_{c_{1}}$ and $H_{c_{2}}$, denote the field values at which the magnetization (a) becomes nonzero and (b) saturates, respectively. Values for $H_{c_{1}}$ and $H_{c_{2}}$ have already been given as Eqs. (7) and (4). $H_{c_{1}}$ has the physical significance that it measures the excitation energy gap between the singlet ground state and the band of lowest-lying excited (triplet) states. To numerical accuracy, the magnetization $M$ depends on $H^{1 / 2}$ near $H_{c_{1}}$ and $H_{c_{2}}$, giving rise to $\frac{1}{2}$-power divergences in the susceptibility.

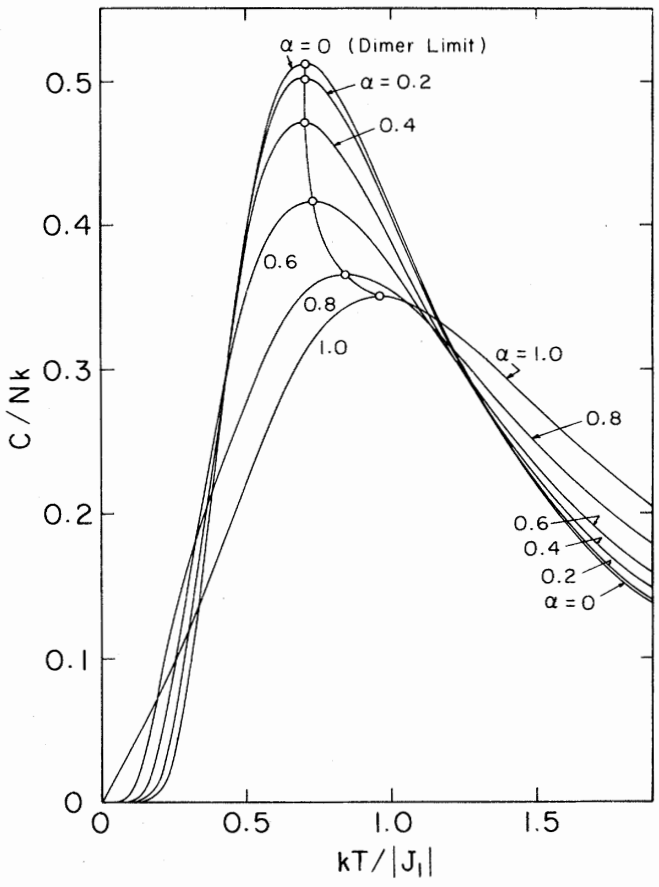

FIG. 4. Zero-field specific-heat curves extrapolated to the thermodynamic limit $(N \rightarrow \infty)$ for alternation values $\alpha=0,0.2,0.4,0.6,0.8$, and 1.0. Curves for $\alpha \neq 1$ show an exponential drop to zero, indicating the presence of an energy gap between the singlet ground state and continua of triplet excited states. Solid line through the open circles shows the locus of specific-heat maxima as a function of alternation.

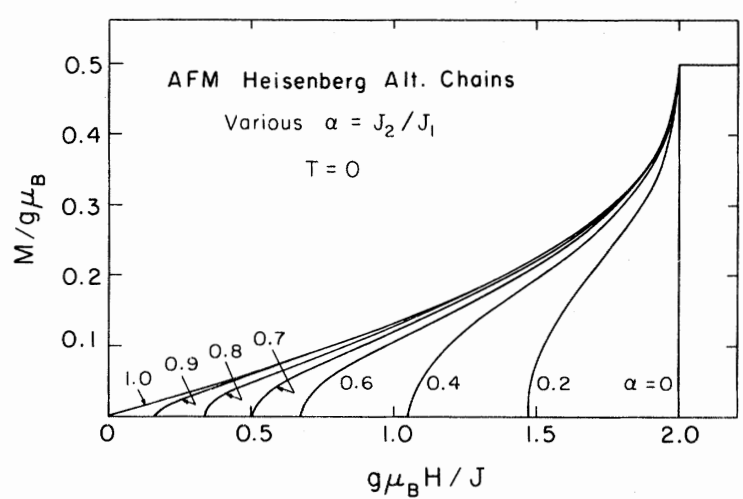

FIG. 5. Zero-temperature magnetization curves for alternating AFM Heisenberg chains as a function of magnetic field for a variety of alternation values. All curves are $N \rightarrow \infty$ extrapolations. For convenience, the magnetic field has been normalized with an exchange parameter $J$ rather than $J_{1}$, where $2 J=J_{1}+J_{2}$. As a result all curves have the same upper critical field, and the behavior with $\alpha$ is more readily apparent. 


\section{LOW-FIELD PROPERTIES}

In order to fit the theoretical alternating-chain curves generated as described in Sec. II to experimental results, we must consider values for three physical parameters: alternation parameter $\alpha$, exchange constant $J$, and $g$ factors, specifically $g_{\|} \equiv g_{b}$ and $g_{\perp}$, where $\|$ and $\perp$ refer to the monoclinic $b$ axis. For an idea of likely values for these parameters, we observe the results of Ajiro et al. ${ }^{17}$ and Diederix et al. $^{29}$ listed in Table I. These parameters have been determined by microscopic methods, namely, paramagnetic $^{17}$ and proton ${ }^{29}$ resonance. Our values for the same parameters will be obtained by comparison of theory and experiment for bulk thermodynamic properties such as the specific heat, magnetic susceptibility, and magnetization. In most cases, the experimental data used in this analysis have been reported in various earlier publications to which the reader is referred for experimental details.

The zero-field specific-heat measurements are the most convenient data set to start the fitting process. The magnitude of the specific-heat maximum depends on $\alpha$ only, i.e., is independent of $J$ and $g$, while the position in temperature of the maximum depends only on the exchange constant $J$. The first point of interest is to see whether the zero-field data are consistent with the independent alternatingchain model, and, in particular, an alternating model with the same $\alpha$ value obtained by Diederix et al. ${ }^{29}$ In Fig. 6 an excellent fit of theory and the experiments of Friedberg and Raquet ${ }^{7}$ is obtained over the whole temperature range of $\sim 0.5-4.2 \mathrm{~K}$. The position of the maximum fixes $J_{1} / k$ at $2.58 \mathrm{~K}$, in agreement with the value $J_{1} / k \sim 2.6 \mathrm{~K}$ of Diederix et al. It may be noticed that a small discrepancy of less than $1 \%$ occurs in the vicinity of the maximum. Apparent improvement could be obtained by increasing $\alpha$ from 0.27 to about 0.3 . However, this would not be realistic for two reasons. First, we are fitting experimental data with a model which ignores the very weak interchain interactions. If such

TABLE I. Comparative table of fitting parameters.

\begin{tabular}{cccc}
\hline \hline & Ajiro et al. $^{\mathrm{a}}$ & Diederix et al. & \\
\hline & & $0.27 \pm 0.02$ & This work \\
\hline$\alpha$ & & $2.60 \pm 0.02$ & $0.27 \pm 0.01$ \\
$J_{1}$ & 2.6 & $2.33 \pm 0.02$ & $2.58 \pm 0.02$ \\
$g_{b}$ & 2.33 & $2.09 \pm 0.02$ & $2.31 \pm 0.03$ \\
$g$ & 2.07 & 2.17 & $2.18 \pm 0.03$ \\
$g_{\text {av }}$ & 2.17 & & \\
\hline \hline
\end{tabular}

a Reference 17.

${ }^{\mathrm{b}}$ Reference 28.

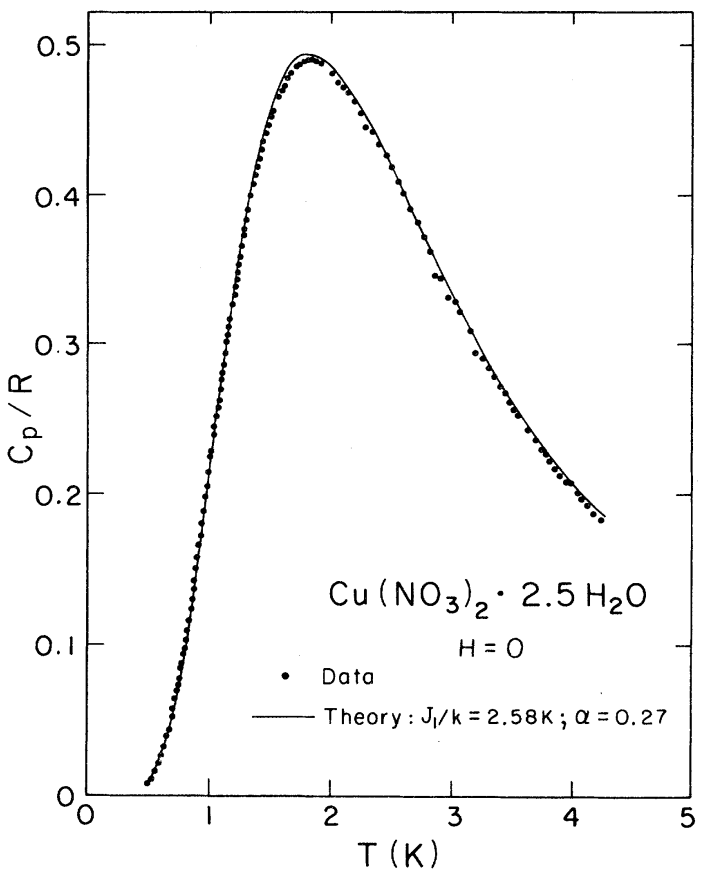

FIG. 6. Heat capacity $C_{p}(H=0)$ of $\mathrm{Cu}\left(\mathrm{NO}_{3}\right) \cdot 2.5 \mathrm{H}_{2} \mathrm{O}$ vs temperature. Data from Friedberg and Raquet (Refs. 7 and 13). Theory as described in text.

interactions could be included in the model, their effect would be to lower slightly the height of the theoretical specific-heat maximum. With this in mind, the present fit at $\alpha=0.27$ should be considered completely satisfactory. A further piece of evidence in support of $\alpha=0.27$ rather than 0.3 comes from the location of the lower critical field $H_{c_{1}}$, which is found to occur at $28.0 \mathrm{kOe}$ from the most sensitive experimental determination, i.e., the proton resonance data. The energy gap $\left(=H_{c_{1}}\right)$ resulting from $\alpha=0.3$ can only be fitted with a rather unacceptably low value of $g_{b}$ (see later discussion of $g$ values). Hence the zero-field specific-heat data confirm the alternation value deduced by Diederix et al. and yield effectively the same value of $J_{1}$ (to within experimental uncertainty).

Next we would like to determine the $g$ values, $g_{b}$ and $g_{\perp}$. This is best done using zero-field singlecrystal susceptibility data. ${ }^{5}$ In Fig. 7 we see the result of fitting theoretical Heisenberg antiferromagnetic alternating-chain curves with $\alpha=0.27$ and $J_{1} / k=2.58 \mathrm{~K}$ to experiment. Note that to within experimental accuracy, the susceptibility measured perpendicular to the $b$ axis is isotropic. Thus the $g$ tensor is essentially axially symmetric although the crystal is monoclinic. An excellent fit both in the helium and in the hydrogen temperature ranges results using the values $g_{b}=2.31$ and $g_{1}=2.11$. 


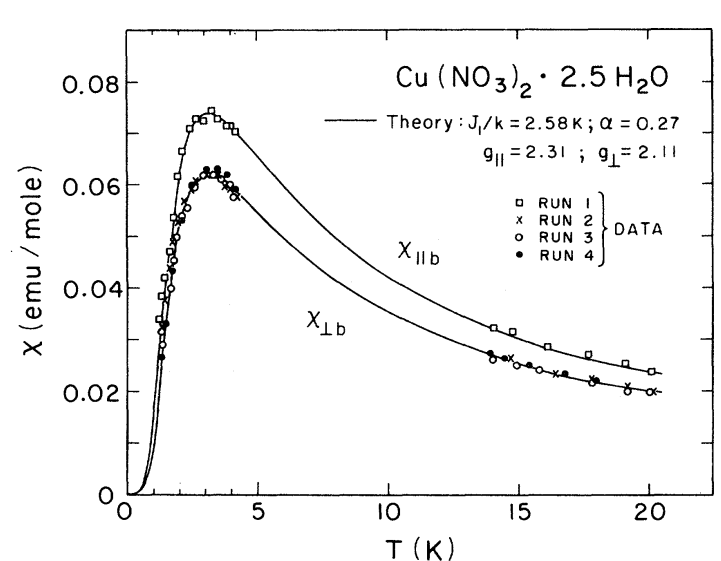

FIG. 7. Low-field ac $(275 \mathrm{~Hz})$ magnetic susceptibility of single-crystal $\mathrm{Cu}\left(\mathrm{NO}_{3}\right)_{2} \cdot 2.5 \mathrm{H}_{2} \mathrm{O}$ measured parallel and perpendicular to the monoclinic $b$ axis, vs temperature. Data from Berger et al. (Ref. 5). Run 1, $\overrightarrow{\mathrm{H}}|| b$ axis, run $2, \overrightarrow{\mathbf{H}} \perp b$ axis and $\perp$ cleavage plane, run $3, \overrightarrow{\mathrm{H}} \perp b$ axis and $\|$ cleavage plane, and run $4, \overrightarrow{\mathrm{H}} \perp b$ axis and at $45^{\circ}$ to cleavage plane. Theory as described in text.

Again, these values are consistent with those obtained by resonance methods and listed in Table I. In Fig. 8 we show the measured ${ }^{5}$ copper nitrate powder susceptibility (including some new data) fitted with the weighted average $g$ value, $g_{\mathrm{av}}=2.18$. This value again agrees with those of Table $I$ and gives an excellent fit to the powder susceptibility over both helium and hydrogen ranges. The specific heat of a powder specimen was also measured in a low applied field of $8.7 \mathrm{kOe}$, giving additional information about $g_{\mathrm{av}}$. In Fig. 9 we show the data fitted to the alternating-chain curve with $\alpha=0.27$, $J_{1} / k=2.58 \mathrm{~K}$, and $g_{\mathrm{av}}=2.18$. The effect of a small

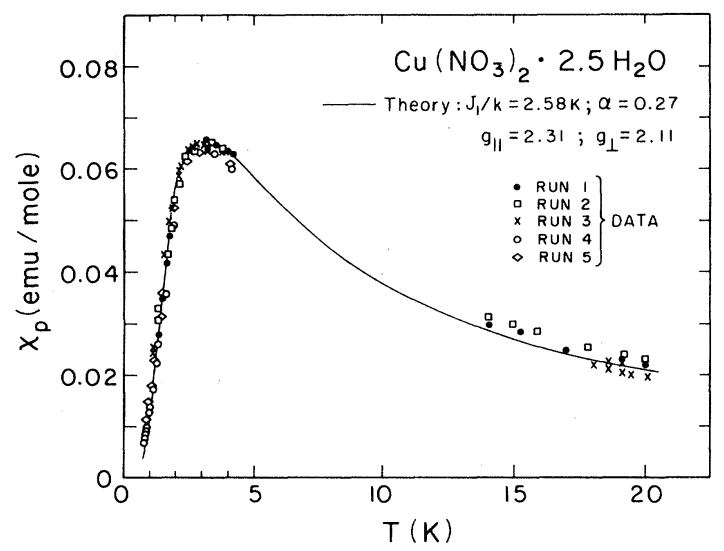

FIG. 8. Low-field ac $(275 \mathrm{~Hz})$ magnetic susceptibility of powdered $\mathrm{Cu}\left(\mathrm{NO}_{3}\right)_{2} \cdot 2.5 \mathrm{H}_{2} \mathrm{O}$ vs temperature. Data runs 1, 2, 4, and 5 from Berger et al. (Ref. 5). Run 3 from this work. Theory as described in text. magnetic field is to depress the height of the specific-heat maximum, and, in Fig. 9, the zero-field theoretical curve is shown dashed, for comparison. An $8 \%$ depression of the height of the maximum is observed. Again, an excellent fit of theory and experiment is obtained at $8.7 \mathrm{kOe}$, to within experimental accuracy.

Magnetization isotherms have also been measured ${ }^{6}$ with fields up to about $40 \mathrm{kOe}$ applied along the $b$ axis at three temperatures: 4.18, 2.031, and $1.278 \mathrm{~K}$. These curves can also be fitted satisfactorily with our theoretical model and the associated parameters, as seen in Fig. 10. It may be observed that while the low-field fit is very good, the theoretical curves are on the low side at the higher fields. This is presumably an example of the fact that the alternating-chain model is better in zero field, and deviations are to be expected at high fields where interchain effects are more apparent. We might note that this high-field discrepancy could be removed by taking account of residual interchain interactions in a mean-field approximation.

The success of the independent alternating-chain model in reproducing the low-field bulk thermodynamic properties of $\mathrm{Cu}\left(\mathrm{NO}_{3}\right)_{2} \cdot 2.5 \mathrm{H}_{2} \mathrm{O}$ is quite striking. The quantitative agreement between the model parameters determined in this process and

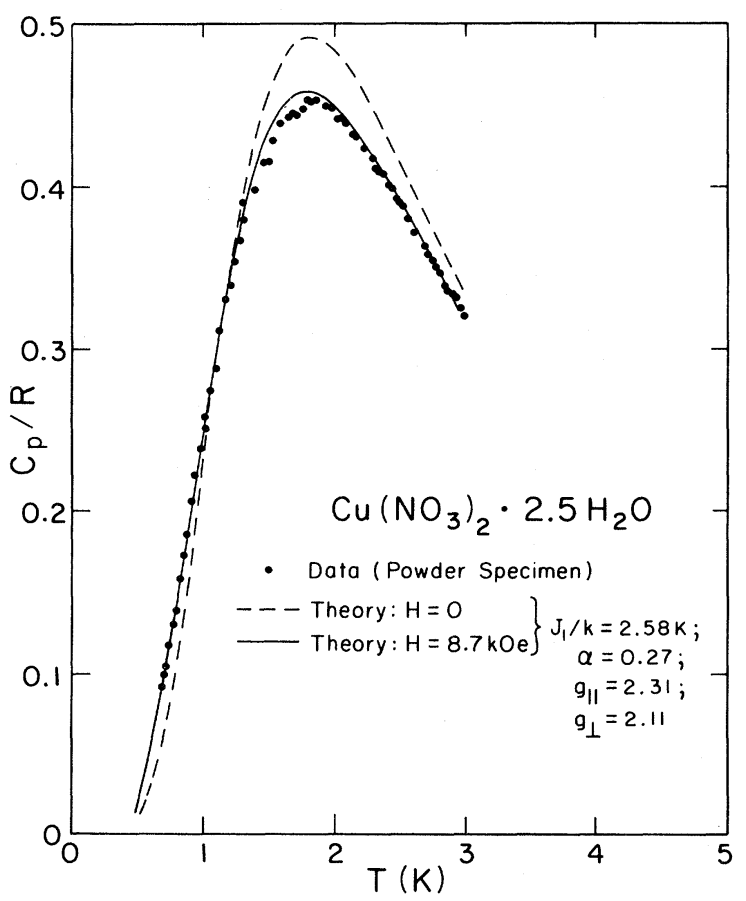

FIG. 9. Heat capacity $C_{p}$ of powdered $\mathrm{Cu}\left(\mathrm{NO}_{3}\right)_{2} \cdot 2.5 \mathrm{H}_{2} \mathrm{O}$ in an applied magnetic field $H=8.7$ kOe vs temperature. Data from Friedberg and Raquet (Refs. 7 and 13). Theory as described in text. 


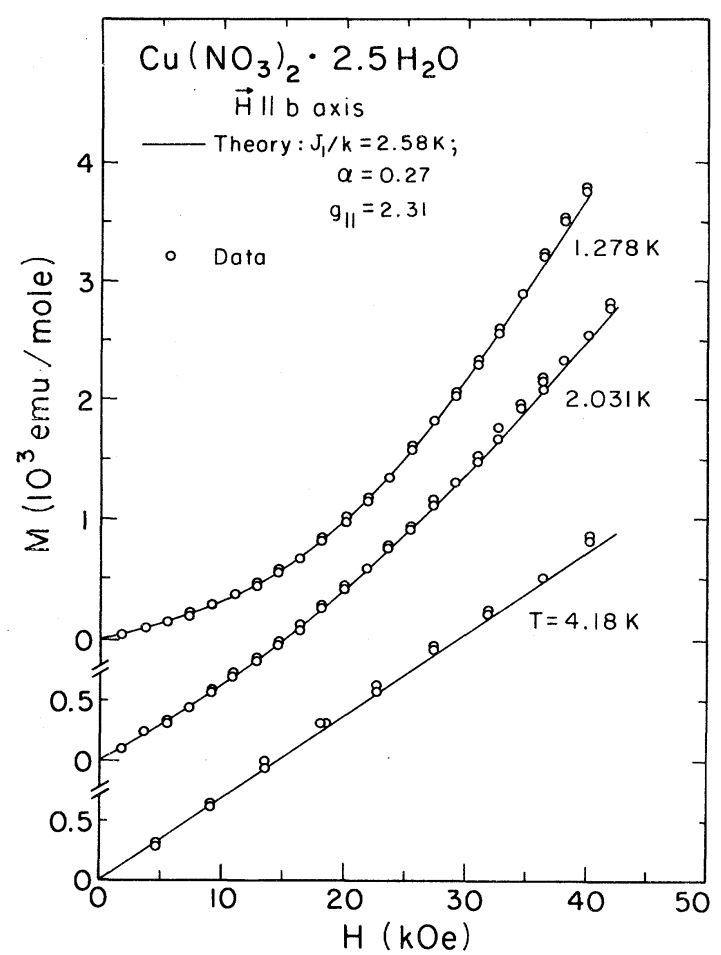

FIG. 10. Magnetization $M$ of single crystal $\mathrm{Cu}\left(\mathrm{NO}_{3}\right)_{2} \cdot 2.5 \mathrm{H}_{2} \mathrm{O}$ vs field $H$ applied along $b$ axis at several temperatures. Data from Myers et al. (Ref. 6). Theory as described in text.

those obtained by other methods suggests that the intrachain interactions in $\mathrm{Cu}\left(\mathrm{NO}_{3}\right)_{2} \cdot 2.5 \mathrm{H}_{2} \mathrm{O}$ are now rather well established, at least within the Heisenberg approximation. These interactions, $J_{1}$ and $J_{2}$, are believed to occur along superexchange pathways involving hydrogen bonds. One might well ask whether substitution of deuterium for hydrogen in this compound significantly alters $J_{1}$ and $J_{2}$, making copper nitrate a good system in which to study the interplay of hydrogen bonding and superexchange.

There is rather clear evidence that the effect of deuteration is certainly not dramatic. Neutron scattering studies ${ }^{12}$ on copper nitrate were actually performed on crystals in which about $90 \%$ of the protons had been replaced by deuterons. No gross crystallographic changes were observed. The fact that the values of $H_{c_{1}}$ and $H_{c_{2}}$ found in the neutron work agree within experimental uncertainty with those observed by proton resonance on hydrated crystals indicates that $J_{1}$ and $J_{2}$ are not greatly altered by deuteration. This is confirmed by some preliminary susceptibility measurements performed on a deuterated crystal in zero field between $\sim 1$ and $4 \mathrm{~K}$. While not very reproducible, presumably because of partial rehydration of the hygroscopic crys- tal between successive runs, these data allow changes in the interaction constants with deuteration of no more than a few percent. Taken at face value, however, they do suggest the occurrence of a detectable isotope effect, further exploration of which might well prove rewarding.

\section{HIGH-FIELD PROPERTIES}

As discussed in Secs. I and II, the high-field behavior of alternating-chain systems is novel and particularly interesting. There are two critical fields: a lower one, $H_{c_{1}}$, and an upper one, $H_{c_{2}}$. Long-range order can occur only with an applied field in the interval $H_{c_{1}}<H<H_{c_{2}}$. In addition, the functional dependence on temperature of the thermodynamic properties of the ideal (independentchain) system varies over the field range $H=0$ to $H>H_{c_{2}}$ in a striking way. Long-range ordering has been observed in $\mathrm{Cu}\left(\mathrm{NO}_{3}\right)_{2} \cdot 2.5 \mathrm{H}_{2} \mathrm{O}$ in fields between $H_{c_{1}} \simeq 28 \mathrm{kOe}$ and $H_{c_{2}} \simeq 43 \mathrm{kOe}$ at temperatures below about $0.17 \mathrm{~K}$ by a variety of techniques. Interchain interaction is thus significant but rather small in comparison with $J_{1}$ and $J_{2}$. This is consistent with the fact that the independent alternating-chain model works well in describing the properties of $\mathrm{Cu}\left(\mathrm{NO}_{3}\right)_{2} \cdot 2.5 \mathrm{H}_{2} \mathrm{O}$ at low field. While we expect the model to fail in the region of the $H$-T diagram where long-range order occurs, it is interesting to see to what extent characteristic features of the independent-chain picture are still evident as the phase boundary is approached from the paramagnetic side.

With this in mind, it is useful to consider some heretofore unpublished heat-capacity data on a composite specimen of coaxially aligned single crystals of $\mathrm{Cu}\left(\mathrm{NO}_{3}\right)_{2} \cdot 2.5 \mathrm{H}_{2} \mathrm{O}$ with a magnetic field applied along the $b$ axis. These measurements were performed in a ${ }^{3} \mathrm{He}-{ }^{4} \mathrm{He}$ dilution cryostat with a $\mathrm{Nb}-\mathrm{Zr}$ superconducting solenoid and a calorimetric insert. Use of a mechanical heat switch made it possible to take data between $\sim 0.08$ and $4.2 \mathrm{~K}$ by the discontinuous heating method. Details of the apparatus, calibration of its thermometer, and the measuring technique have been given elsewhere. ${ }^{59,60}$ Measurements on a $3.00-\mathrm{g}$ specimen in zero field, corrected for small addenda contributions, yielded results in good agreement between 0.6 and $4.2 \mathrm{~K}$ with the earlier data ${ }^{7}$ on a large powder sample shown in Fig. 6. The same addenda correction was applied to the infield data taken with a 5.08-g sample. The lattice contribution is quite small over most of the temperature range.

We limit our discussion to the heat-capacitytemperature curves for two values of $\overrightarrow{\mathrm{H}}|| b$, namely, 
28.2 and $35.7 \mathrm{kOe}$. The lower of these fields is very close to the lower critical field $H_{c_{1}}$. The larger field lies between $H_{c_{1}}$ and $H_{c_{2}}$ and corresponds roughly to the field at which $\mathrm{Cu}\left(\mathrm{NO}_{3}\right)_{2} \cdot 2.5 \mathrm{H}_{2} \mathrm{O}$ exhibits longrange order at the highest temperatures with $\overrightarrow{\mathrm{H}} \| b$. Figure 11 shows the heat capacity of $\mathrm{Cu}\left(\mathrm{NO}_{3}\right)_{2} \cdot 2.5 \mathrm{H}_{2} \mathrm{O}$ between 0.08 and $4.4 \mathrm{~K}$ with $H_{b}=28.2 \mathrm{kOe}$. $C_{p}$ exhibits rounded maxima at 0.47 and $2.34 \mathrm{~K}$ and no evidence down to the lowest temperature of even a small cooperative peak associated with long-range ordering. The solid curve, calculated for the independent alternating chain with the parameters determined from low-field data, is seen to give very satisfactory agreement with the measurements. Convergence problems have limited these calculations to temperatures above $0.25 \mathrm{~K}$. The dashed segment represents a reasonable extrapolation to $T=0$. The double maximum in $C_{p}$ corresponds to the bunching of levels of a strongly alternating antiferromagnet in an applied field mentioned in Sec. II. The fact that there are two maxima can be understood qualitatively by imagining the alternation taken to the limit $\alpha=0$. In that case we have a system of isolated dimers each with a singlet ground state separated by an energy $2\left|J_{1}\right| / k \simeq 5.2$ $\mathrm{K}$ from an excited triplet. In a field of $28.2 \mathrm{kOe}$ the triplet splits, giving a first excited level $\sim 0.8 \mathrm{~K}$ above the ground state, a second at $5.2 \mathrm{~K}$, and a third at $\sim 9.6 \mathrm{~K}$. The resulting heat-capacity curve for a system of dimers is a Schottky anomaly with two fully resolved maxima at roughly the temperatures found experimentally. As $\alpha$ is increased to 0.27 , the density of states of the alternating chain exhibits maxima and minima generically related to those of the spin pair.

Another significant feature of Fig. 11 is the limit-

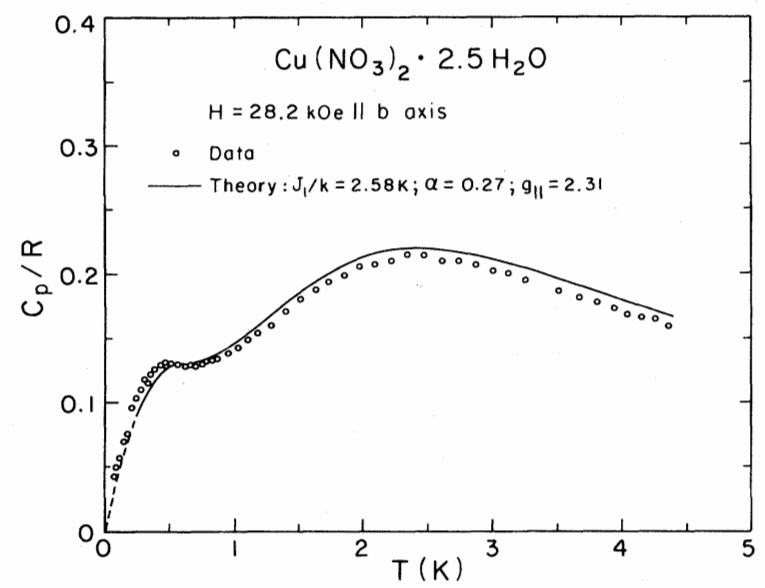

FIG. 11. Heat capacity $C_{p}$ of composite single-crystal specimen of $\mathrm{Cu}\left(\mathrm{NO}_{3}\right)_{2} \cdot 2.5 \mathrm{H}_{2} \mathrm{O}$ vs temperature with a field $H=28.2 \mathrm{kOe}$ applied along the $b$ axis. ing behavior of $C_{p}$ as $T \rightarrow 0$. As indicated in Sec. II, Eqs. (11) and (12), theory predicts for the antiferromagnetic alternating chain that when $H=H_{c_{1}}$ and $H_{c_{2}}$ the heat capacity $C_{H} \propto T^{1 / 2}$ in the lowtemperature limit. The experimental data and the calculated theoretical curve of Fig. 11 are quite consistent with such behavior at the lowest temperatures to which they have been extended. In general, the independent-chain model gives an excellent description of the heat capacity of $\mathrm{Cu}\left(\mathrm{NO}_{3}\right)_{2} \cdot 2.5 \mathrm{H}_{2} \mathrm{O}$ in a field $H \simeq H_{c_{1}}$. The maximum discrepancy between theory and experiment is about $4 \%$ and is probably attributable primarily to the neglect of residual interchain interactions.

The results of measurements in an applied field $H_{b}=35.7 \mathrm{kOe}$ between 0.1 and $4.6 \mathrm{~K}$ are shown in Fig. 12(a). The data for $T<1 \mathrm{~K}$ are plotted on an expanded temperature scale in Fig. 12(b) to bring out more clearly the $\lambda$-like cooperative anomaly marking the onset of long-range order at $T_{c} \simeq 0.16$ K. Figure 12(b) also shows the well-resolved rounded maximum near $\sim 0.25 \mathrm{~K}$ corresponding to the growth of substantial short-range order in the chainlike spin arrays. Both these features of the heat capacity with $H_{b}=35.7$ kOe have been previously reported by van Tol et al., ${ }^{19}$ whose data were limited to $T<0.8 \mathrm{~K}$ because of the use of a superconducting heat switch. In Fig. 12(a), however, we see that there is an additional rounded maximum at $2.6 \mathrm{~K}$ which is also attributable to the alternating-chain system in $\mathrm{Cu}\left(\mathrm{NO}_{3}\right)_{2} \cdot 2 \cdot 5 \mathrm{H}_{2} \mathrm{O}$. Neglect of interchain coupling is a more drastic approximation than before, since $H=35.7 \mathrm{kOe}$ is in the field range in which long-range order occurs. While our independent-chain model cannot account for the cooperative $\lambda$ anomaly at $T_{c}$, it does, however, predict two rounded maxima very much like those found experimentally. The heavy solid curve in Fig. 12(a) has been calculated using, as before, the model parameters determined from zero-field data. The lower of the two maxima is observed at about $0.25 \mathrm{~K}$, in fair agreement with the theoretical result. It should be pointed out, however, that theoretically convergence is slow for this maximum, with oscillatory character for $N=4,6,8$, and 10 . Therefore, we expect the curves for $N=8$ and 10 to bracket the limiting result for which our calculated (heavy) curve should be a good approximation. Theoretically we estimate $C / R$ at the lower maximum to be $\sim 0.2$, which is somewhat higher than the experimental value of $\sim 0.167$. However, it should be borne in mind that the ordering peak at $T_{c}$ will "remove" entropy from the alternating-chain anomaly above it so that such a discrepancy is, in fact, to be expected. Allowing for this effect, the agreement of 


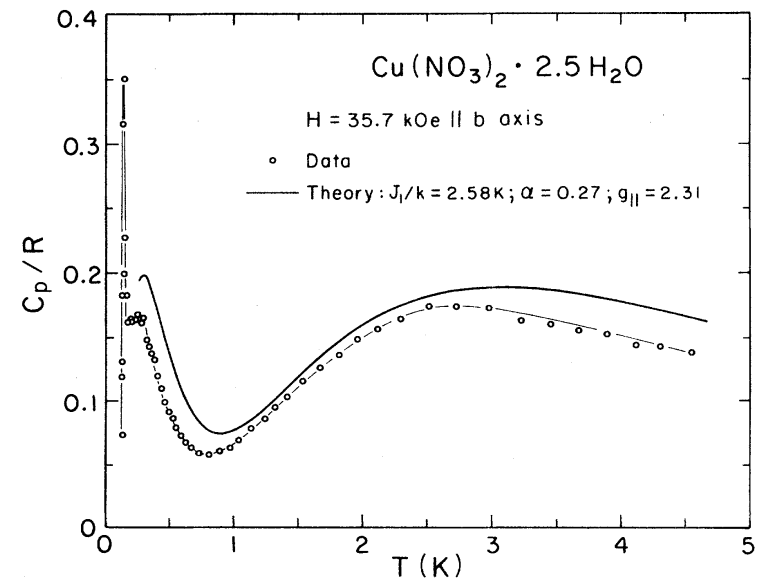

(a)

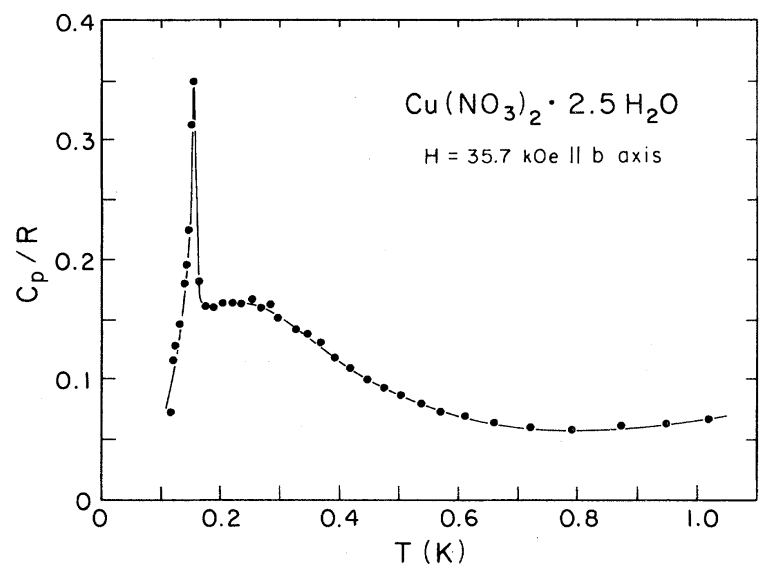

(b)

FIG. 12. Heat capacity $C_{p}$ of composite single-crystal specimen of $\mathrm{Cu}\left(\mathrm{NO}_{3}\right)_{2} \cdot 2.5 \mathrm{H}_{2} \mathrm{O}$ vs temperature with a field $H=35.7 \mathrm{kOe}$ applied along the $b$ axis: (a) full temperature range with light curve shown to aid the eye and theoretical (heavy) curve as described in text; (b) expanded temperature scale with curve shown to aid the eye.

the independent-chain model and experiment may be considered quite satisfactory at the lower maximum and at temperatures up to about $2.5 \mathrm{~K}$. At about 2.6 $\mathrm{K}$, the experimental points pass through their upper maximum, while the theoretical curve does not do so until $3.1 \mathrm{~K}$. In this region, the theoretical calculations converge rapidly, and, since the ordering temperature is now relatively remote, we might expect better agreement with experiments than at the lower maximum. This does not seem to be the case even though one has no quantitative estimate of the effect of the interchain interactions in either region. We hesitate, however, to attach much significance to this apparent discrepancy, since the uncertainty in the experimental results may be rather large due to uncertainties in a number of corrections which should be applied to the data. Among these we may mention allowance for the effect of the magnetic field on the thermometer resistance and the addenda correction which becomes significant at the higher temperature. Furthermore, it should be noted that the demagnetizing field inside the composite specimen is difficult to estimate with reasonable accuracy so that we have not attempted such a correction of the data. Thus the internal field may not only be somewhat different from the applied field, but slightly nonuniform as well.

Further study of the region between the two critical fields, both theoretical and experimental, would be desirable. For example, while the magnetic thermodynamic properties are expected to show the same functional dependence on $T$ at both critical fields, asymmetry effects observable experimentally in, say, cooling isentropes ${ }^{29}$ and adiabatic susceptibilities, ${ }^{29}$ and also in theoretical studies on alternating $X Y$ chains, ${ }^{61}$ imply that the amplitudes at $H_{c_{1}}$ and $H_{c_{2}}$ differ. An estimate of at least the ratio of the amplitudes might be obtained from a specificheat study at about $44 \mathrm{kOe}$, the upper critical field.

\section{v. DISCUSSION}

Copper nitrate is a novel system; an antiferromagnetic Heisenberg strongly alternating chain whose magnetic characteristics are such that the entire phase region in $T$ and $H$ can conveniently be explored experimentally. We have demonstrated here an extraordinarily good fit between theory and an extensive collection of low- and high-field magnetic thermodynamic experiments. We have used a theoretical alternating-chain model with alternation ratio $\alpha=0.27$ and exchange constant $J_{1} / k=2.58$ $\mathrm{K}$, with $g_{b}=2.31$ and $g=2.11$. In Table I we indicate estimates of uncertainties assigned to these parameters. The uncertainties for $\alpha$ and $J_{1}$ are probably conservative. Small residual discrepancies between theory and experiment are systematically attributable qualitatively to weak interchain coupling and make attempts to refine these estimates not very meaningful. The exceptional magnetic characterization of this system suggests its use for further experimental study, particularly in the vicinity of the high-field ordering region between 28 and 44 kOe.

However, it seems fair to point out that many of the essential characteristics of copper nitrate were inferred previously from more primitive investigations. For example, a dimer model with weak anti- 
ferromagnetic interdimer interactions used to fit magnetization data, gave $J_{1} / k=2.57 \mathrm{~K}$ and $g_{b}=2.35 .^{6,14}$ A six-spin alternating cluster gave a simultaneous fit to all low-field data and magnetization isotherms with $J_{1} / k=2.59 \mathrm{~K}$ and $g_{b}=2.29$. (However, the alternation value required was somewhat high at $\alpha=0.36$.) Hence, the more sophisticated theory and experiments featured herein have largely confirmed earlier conjectures. ${ }^{4,15}$ In particular, a prediction that the fitting parameters $(J$ and $g$ ) derived from a six-spin cluster model will differ from those corresponding to an infinitely long sys- tem by only about $2 \%$ (Ref. 14) has been borne out.

\section{ACKNOWLEDGMENTS}

This work was supported in part by National Science Foundation under Grant No. DMR81-06491 and in part by NSF Grant No. DMR80-10819. One of us (J.C.B.) gratefully acknowledges receipt of NATO Grant No. 1338, which made possible extended visits to the Kamerlingh Onnes Laboratorium, Leiden, to which the authors are indebted for the provision of computer time. J.C.B.'s contribution to this work was begun while at the Physics Department, Carnegie-Mellon University.
*Permanent address: Department of Chemistry, Faculty of Science, Kyoto University, Kyoto, Japan.

${ }^{\dagger}$ Present address: Westinghouse Research Laboratories, Beulah Road, Pittsburgh, PA 15235.

$\ddagger$ Present address: The Technical University of Delft, Delft, Netherlands.

${ }^{1}$ See review by, P. L. Nordio, Z. G. Soos, and H. M. McConnell, Annu. Rev. Phys. Chem. 17, 237 (1966); Z. G. Soos, J. Chem. Phys. 46, 4284 (1967).

${ }^{2}$ I. S. Jacobs, J. W. Bray, H. R. Hart, Jr., L. V. Interrante, J. S. Kasper, G. D. Watkins, D. E. Prober, and J. C. Bonner, Phys. Rev. B 14, 3036 (1976); J. W. Bray, L. V. Interrante, I. S. Jacobs, and J. C. Bonner, in Extended Linear Chain Materials, edited by J. S. Miller (Plenum, New York) (in press).

${ }^{3}$ See review by W. E. Hatfield, J. Appl. Phys. 2ㅡ, 1985 (1981).

4J. J. Smit, L. J. de Jongh, J. A. C. van Ooijen, J. Reedijk, and J. C. Bonner, Physica B 97, 229 (1979).

${ }^{5}$ L. Berger, S. A. Friedberg, and J. T. Schriempf, Phys. Rev. 132,1057 (1963).

6B. E. Myers, L. Berger, and S. A. Friedberg, J. Appl. Phys. 40, 1149 (1969).

${ }^{7}$ S. A. Friedberg and C. A. Raquet, J. Appl. Phys. 39, 1132 (1968).

8J. Garaj, Acta Chem. Scand. 22, 1710 (1968).

${ }^{9}$ B. Morosin, Acta Crystallogr. B 26, 1203 (1970).

10S. Wittekoek and N. J. Poulis, J. Appl. Phys. 39, 1017 (1968).

${ }^{11} \mathrm{~K}$. Amaya, Y. Tokunaga, R. Yamada, Y. Ajiro, and T. Haseda, Phys. Lett. A $\underline{28}, 732$ (1969).

12J. Eckert, D. E. Cox, G. Shirane, S. A. Friedberg, and H. Kobayashi, Phys. Rev. B 20, 4596 (1979).

${ }^{13}$ See Ref. 7. Note that all $C_{p}$ data point in Figs. 1 and 2 of this paper must be multiplied by a factor of 0.963 to compensate for the fact that the formula weight used in the reduction of the data was inadvertently taken to be that of $\mathrm{Cu}\left(\mathrm{NO}_{3}\right)_{2} \cdot 3 \mathrm{H}_{2} \mathrm{O}$ instead of $\mathrm{Cu}\left(\mathrm{NO}_{3}\right)_{2} \cdot 2.5 \mathrm{H}_{2} \mathrm{O}$. When this is done, the discrepancy with the dimer model is quite evident.

${ }^{14} \mathrm{~A}$ review of early experimental and theoretical work on copper nitrate is contained in an unpublished report by J. C. Bonner, Carnegie-Mellon University, July 1970.

15J. C. Bonner, S. A. Friedberg, H. Kobayashi, and B. E. Myers, in Proceedings of the Twelfth International Conference on Low Temperature Physics, Kyoto, 1970, edited by E. Kanda (Keigaku, Tokyo, 1971), p. 691.

16J. C. Bonner and S. A. Friedberg, in Magnetism and Magnetic Materials-1973 (Boston), Proceedings of the 19th Annual Conference on Magnetism and Magnetic Materials, edited by C. D. Graham and J. J. Rhyne (AIP, New York, 1974), p. 1311.

${ }^{17}$ Y. Ajiro, N. S. VanderVen, and S. A. Friedberg, in Proceedings of the Thirteenth International Conference on Low Temperature Physics, Boulder, 1973, edited by K. D. Timmerhaus, W. J. O'Sullivan, and E. F. Hammel (Plenum, New York, 1974), p. 380.

${ }^{18}$ M. W. van Tol, L. S. J. M. Henkens, and N. J. Poulis, Phys. Rev. Lett. 27, 739 (1971).

${ }^{19}$ M. W. van Tol, K. M. Diederix, and N. J. Poulis, Physica (Utrecht) 64, 363 (1973).

${ }^{20}$ M. W. van Tol, M. Matsuura, and N. J. Poulis, J. Phys. (Paris) Colloq. C1 Suppl. 2-3, 32, C1-943 (1971).

${ }^{21}$ M. W. van Tol, M. H. C. Eijkelhof, and A. J. van Duyneveldt, Physica 60, 223 (1972).

${ }^{22}$ M. W. van Tol, thesis, University of Leiden, 1972 (unpublished).

${ }^{23}$ Y. Tokunaga, S. Ikeda, J. Watanabe, and T. Haseda, J. Phys. Soc. Jpn. 32, 429 (1972).

${ }^{24}$ K. Amaya, N. Yamashita, M. Matsuura, and T. Haseda, J. Phys. Soc. Jpn. 37, 1173 (1974).

${ }^{25}$ K. M. Diederix, J. P. Groen, and N. J. Poulis, Physica (Utrecht) B $\underline{86-88}, 1151$ (1977).

${ }^{26}$ K. M. Diederix, J. P. Groen, and N. J. Poulis, Phys. Lett. 60A, 247 (1977).

${ }^{27}$ K. M. Diederix, J. P. Groen, L. S. J. M. Henkens, T. O. Klaassen, and N. J. Poulis, Physica (Utrecht) B $\underline{93}, 99$ (1978).

${ }^{28}$ K. M. Diederix, J. P. Groen, L. S. J. M. Henkens, T. O. Klaassen, and N. J. Poulis, Physica (Utrecht) B $\underline{94}, 9$ (1978).

${ }^{29}$ K. M. Diederix, H. W. J. Blöte, J. P. Groen, T. O. 
Klaassen, and N. J. Poulis, Phys. Rev. B 19, 420 (1979).

${ }^{30}$ K. M. Diederix, thesis, University of Leiden, 1979 (unpublished).

${ }^{31}$ T. Haseda, Y. Tokunaga, R. Yamada, Y. Kuramitsu, S. Sakatsume, and $\mathrm{K}$. Amaya, in Proceedings of the Twelfth International Conference on Low Temperature Physics, Kyoto, 1970, Ref. 15, p. 685.

${ }^{32}$ M. Tachiki and T. Yamada, J. Phys. Soc. Jpn. 28, 1413 (1970).

${ }^{33}$ M. Tachiki, T. Yamada, and S. Maekawa, J. Phys. Soc. Jpn. 29, 656 (1970).

${ }^{34}$ M. Tachiki and T. Yamada, Prog. Theor. Phys. Suppl. 46, 291 (1970).

${ }^{35}$ M. Tachiki, T. Yamada, and S. Maekawa, J. Phys. Soc. Jpn. 29, 663 (1970).

36J. C. Bonner, S. A. Friedberg, and B. E. Myers, Bull. Am. Phys. Soc. 16, 327 (1971).

37J. C. Bonner and S. A. Friedberg, in Proceedings of the Conference on Phase Transitions and Their Applications in Materials Science, edited by H. K. Henisch, R. Roy, and L. E. Cross (Pergamon, New York, 1973), p. 429.

38J. C. Bonner and H. W. J. Blöte, Phys. Rev. B $\underline{25}, 6959$ (1982).

${ }^{39}$ W. Duffy, Jr. and K. P. Barr, Phys. Rev. $\underline{165}, 647$ (1968).

${ }^{40} \mathrm{M}$. Drawid and J. W. Halley in Magnetism and Magnetic Materials-1976 (Joint MMM-Intermag Conference, Pittsburgh), Partial Proceedings of the First Joint MMM-Intermag Conference, edited by J. J. Becker and G. H. Lander (AIP, New York, 1976), p. 208.

${ }^{41}$ J. C. Bonner, H. W. J. Blöte, and J. D. Johnson, J. Appl. Phys. 50, 7379 (1979).

42J. des Cloizeaux and M. Gaudin, J. Math. Phys. 7, 1384 (1966). For corrected excitations, see J. D. Johnson and B. M. McCoy, Phys. Rev. A $\underline{6}, 1613$ (1972).

${ }^{43}$ A. B. Harris, Phys. Rev. B ㄱ, 3166 (1973).
${ }^{44}$ N. D. Mermin and H. Wagner, Phys. Rev. Lett. 17, 1133 (1966).

45J. C. Bonner, Bull. Am. Phys. Soc. 19, 305 (1974).

46J. C. Bonner and J. F. Nagle, J. Appl. Phys. 42, 1280 (1971).

${ }^{47}$ J. Bernasconi and F. Rys, Phys. Rev. B 4 3045 (1971).

${ }^{48}$ F. Y. Wu (unpublished).

49J. C. Bonner and M. E. Fisher, Phys. Rev. 135, A640 (1964).

50J. C. Bonner, J. Appl. Phys. 49, 1299 (1978).

${ }^{51}$ R. J. Baxter, J. Stat. Phys. 9, 145 (1973).

${ }^{52}$ E. Lieb and D. Mattis, J. Math. Phys. $\underline{3}, 749$ (1962).

53J. L. Black and V. J. Emery, Phys. Rev. B 23, 429 (1981).

${ }^{54}$ M. Kohmoto, M. den Nijs, and L. P. Kadanoff, Phys. Rev. B 24, 5229 (1981).

55J. C. Bonner, H. W. J. Blöte, J. W. Bray, and I. S. Jacobs, J. Appl. Phys. 50, 1810 (1979).

56 Our extrapolations have already been published in Refs. 57 and 58 below.

${ }^{57}$ L. J. de Jongh, in Recent Developments in Condensed Matter Physics, Proceedings of the 1980 Annual Conference of the Condensed Matter Division of the European Physical Society, edited by J. J. Devreese (Plenum, New York, 1981), Vol 1, p. 343.

58J. C. Bonner, H. W. J. Blöte, H. Beck, and G. Müller, Physics in One Dimension, Vol. 23 of Springer Series in Solid State Sciences, edited by J. Bernasconi and T. Schneider (Springer, Berlin, 1981), p. 115.

${ }^{59}$ M. Karnezos, D. L. Meier, and S. A. Friedberg, Phys. Rev. B 17, 4375 (1978).

${ }^{60}$ D. L. Meier, Doctoral thesis, Carnegie Mellon University, 1975 (unpublished).

${ }^{61}$ J. H. H. Perk, H. W. Capel, Th.J. Siskens, and M. J. Zuilhof, Physica (Utrecht) A $\underline{81}, 319$ (1975). 